\title{
A assistência no pré-natal no contexto da estratégia de saúde da família sob o olhar do enfermeiro
}

\author{
Prenatal assistance in the context of the family health strategy under the nurse's \\ perception
}
Asistencia prenatal en el contexto de la estrategia de salud de la familia bajo la mirada del enfermero

Amanda Quadros de Souza ${ }^{1 *}$, Mara Regina Caino Teixeira Marchiori ${ }^{1}$, Fernanda Beheregaray Cabral$^{2}$, Claudia Maria Diaz ${ }^{1}$, Naiana Oliveira dos Santos ${ }^{1}$, Ana Laura Zuchetto Pizolotto 1.

\section{RESUMO}

Objetivo: Conhecer a percepção de enfermeiras acerca da assistência e das orientações durante o pré-natal em relação ao parto no contexto da Estratégia de Saúde da Família. Metodologia: Trata-se de um estudo exploratório e descritivo, o qual foi estruturado a partir de uma abordagem qualitativa, realizado no período entre março a agosto de 2018, por meio de entrevista semiestruturada com quatro enfermeiras que atuavam nas Estratégias de Saúde da Família de um município do interior do Rio Grande do Sul. Resultados: Com base na análise de conteúdo, os dados resultaram em duas categorias temáticas: Orientações durante a consulta de pré-natal; e percepção das enfermeiras sobre o cuidado ofertado durante o pré-natal nas Estratégias de Saúde da Família. Os resultados mostraram que as enfermeiras não abordavam o parto como tema das consultas de pré-natal. Considerações finais: A percepção da assistência prestada pelas enfermeiras refletiu sobre o seu saber fazer no atendimento à gestante durante o pré-natal. $O$ enfermeiro dentro das Estratégias de Saúde da Família foi visto com muitas atribuições, fazendo com que se repense a atenção dada as gestantes durante o pré-natal, visto que é um momento que a mulher necessita de informações de qualidade.

Palavras-chave: Estratégia de Saúde da Família, Parto, Cuidado Pré-natal, Enfermagem materno-infantil.

\begin{abstract}
Objective: Was to know the nurses' perception about care and guidance during prenatal care in relation to childbirth in the context of the Family Health Strategy. Methodology: This is an exploratory and descriptive study, which was structured based on a qualitative approach, carried out in the between March and August of 2018, through interviews half structured with four nurses who worked in the Strategies Family Health of a county in the countryside of Rio Grande do Sul. Results: Based on the content analysis, the data resulted in two thematic categories: Guidance during the prenatal visit; and nurses' perception about the care offered during prenatal care in the Family Health Strategies. Guidance on the timing of childbirth did not appear among the topics addressed during the prenatal visit. Final considerations: The perception of the care provided by the nurses of the Family Health Strategies reflected on their knowhow about the treatment of pregnant women during prenatal care. The nurse within the Family Health Strategies was seen with many attributions, causing the attention given to pregnant women during the prenatal period, since it is a moment when the woman needs quality information.
\end{abstract}

Keywords: Family Health Strategy; Childbirth; Prenatal care; Maternal-Child Nursing.

\footnotetext{
${ }^{1}$ Universidade Franciscana (UFN), Santa Maria, RS. *E-mail: amandaqsouza@hotmail.com 2Universidade Federal de Santa Maria (UFSM), Santa Maria, RS.
} 


\section{RESUMEN}

Objetivo: Conocer la percepción de enfermeras acerca de la asistencia y de las orientaciones durante el prenatal en relación al parto en el contexto de la Estrategia de Salud de la Familia. Metodología: Se trata de un estudio exploratorio y descriptivo, el cual fue estructurado a partir de un abordaje cualitativo, realizado en el período entre los meses de marzo a agosto de 2018, por medio de entrevistas semiestructurada con cuatro enfermeras que actuaban que actuaban en las Estrategias de Salud de la Familia de un municipio del interior de Rio Grande do Sul. Resultados: Con base en el análisis de contenido, los datos resultaron en dos categorías temáticas: Orientaciones durante la consulta de prenatal; y percepción de las enfermeras sobre el cuidado ofrecido durante el prenatal en las Estrategias de Salud de la Familia. Los resultados mostraron que las enfermeras no abordaban el parto como tema de las consultas de prenatal. Consideraciones finales: La percepción de la asistencia prestada por las enfermeras de las Estrategias de Salud de la Familia reflexionó acerca de su atención a la gestante durante el prenatal. El enfermero dentro de las Estrategias de Salud de la Familia fue visto con muchas atribuciones, haciendo que la atención dada a las gestantes se repita durante el período prenatal, ya que las mujeres necesitan información de calidad.

Palabras clave: Estrategia de Salud de la Familia; entrega; Cuidado prenatal; Enfermería Maternoinfantil.

\section{INTRODUÇÃO}

O pré-natal é a assistência prestada à gestante desde a concepção, visando à saúde adequada da mulher e do feto, evitando doenças que podem afetar o binômio durante a gestação e proporcionando gravidez e nascimento saudáveis (SILVA MB e MONTEIRO OS, 2010).

O eixo estruturante da atenção básica é a Estratégia de Saúde da Família (ESF), a qual propõe, principalmente, prevenção e diagnóstico precoce, assistindo integralmente à população, sendo a equipe mínima composta por enfermeiro, médico, técnico de enfermagem e agentes comunitários de saúde (ACS) (BRASIL, 2017).

A consulta de pré-natal de baixo risco na atenção básica pode e deve ser realizada pelo enfermeiro, segundo o Ministério da Saúde (MS) e conforme direito garantido pela Lei do Exercício Profissional da Enfermagem, regulamentada pelo Decreto $n^{\circ} .94 .406$, de 8 de junho de 1987. Assim sendo, essa classe tem um importante papel no cuidado à gestante, à puérpera e ao recém-nascido, pois é nesses períodos que a mulher muitas vezes se sente insegura e precisa de apoio (RAGAGNIN MV et al., 2017; BRASIL, 2012).

Segundo o MS, durante o pré-natal, o enfermeiro, através de seu conhecimento e prática, atuará orientando a gestante e sua família com informações sobre seus medos e fantasias em relação ao parto, preparo para o momento do parto, orientações e incentivo para o parto normal - resgatando-o como um processo fisiológico, sinais e sintomas do parto - direitos da parturiente, orientações e incentivo para a amamentação, entre outras informações pertinentes (BRASIL, 2012a).

Deste modo, abordar as percepções das gestantes sobre a assistência pré-natal prestada pelo enfermeiro se faz necessária para o fortalecimento das ações realizadas durante o seguimento das consultas, visando a promoção da saúde e contribuindo para a garantia dos direitos (RAMOS ASMB, et al., 2018).

Estudos demonstram que as gestantes que realizam o pré-natal com enfermeiros, declaram-se satisfeitas com as consultas, devido à forma como se estabelecem as relações de comunicação, na qual o acolhimento e a escuta são privilegiados. O enfermeiro tem fundamental importância na assistência pré-natal, entretanto, são necessários investimentos em sua qualificação, para que as consultas possam ser realizadas da melhor forma possível (ARAUJO SM, et al., 2010).

Considerando as características preconizadas para a assistência pré-natal, percebe-se que o cuidado com a gestação configura um dos principais indicadores do prognóstico do nascimento. Com base no exposto, objetivou-se conhecer a percepção de enfermeiras acerca da assistência e das orientações ofertadas durante o pré-natal em relação ao parto no contexto da ESF. 


\section{MÉTODO}

Trata-se de um estudo exploratório e descritivo, estruturado a partir de uma abordagem qualitativa. Fizeram parte do estudo quatro enfermeiras que atuavam nas ESFs de um município localizado no interior do Rio Grande do Sul.

O critério de inclusão utilizado foi atuar há no mínimo um ano na ESF, enquanto o de exclusão foi estar afastado, de férias ou não querer participar da pesquisa. Ao todo, 4 enfermeiras participaram do estudo. As participantes foram selecionadas conforme os critérios de inclusão.

A coleta de dados foi realizada nos meses de março a agosto de 2018, mediante contato prévio via telefone, sendo que as entrevistas foram agendadas de forma individual de acordo com a disponibilidade de cada sujeito. Realizou-se entrevista individual, com base em questões norteadoras, o que possibilitou a interação entre a pesquisadora e entrevistada. As entrevistas foram realizadas nas ESFs, em salas privadas, foram gravadas com o consentimento das participantes para que se obtivesse um material autêntico.

É importante ressaltar que, anteriormente à coleta de dados do presente estudo, foram realizadas duas entrevistas pilotos com sujeitos que não participariam da pesquisa, mas que se assemelhariam com os participantes desta. Optou-se por realizar o teste com enfermeiras de UBS, a fim de validar o instrumento de pesquisa que seria usado com os sujeitos delimitados para este estudo.

Após a organização, os dados foram interpretados com base na análise de conteúdo temática, sistematizada em três etapas. Na primeira etapa, denominada de pré-análise, foi realizada a leitura exaustiva dos dados encontrados, seguida da organização do material e da formulação de hipóteses. Dando seguimento, foi realizada a exploração do material. Na terceira e última fase da análise, os dados foram interpretados e delimitados conforme eixos temáticos a partir da compreensão dos significados estabelecidos (BARDIN L, 2011).

O projeto foi submetido e aprovado pelo Comitê de Ética em Pesquisa (CEP) da Universidade Franciscana, sob o número 1.527.515. Os participantes tiveram clareza quanto aos objetivos da pesquisa, por meio da leitura e assinatura do Termo de Consentimento Livre e Esclarecido (TCLE), conforme resolução do Conselho Nacional de Saúde 466/12 (BRASIL, 2012b).

Destaca-se que os nomes das participantes foram mantidos anônimos para preservar as suas identidades. No texto, as falas foram identificadas por E1, E2 e assim sucessivamente.

\section{RESULTADOS E DISCUSSÃO}

Inicialmente, foi realizado a caracterização dos participantes investigados como a possibilidade de uma melhor visualização do grupo em estudo (Tabela 1).

Tabela 1 - Caracterização dos participantes.

NOME SEXO IDADE

TEMPO PÓSFORMAÇÃO GRADUAÇÃO

E1

E2

E3

E4

SEXO

IDADE

29 anos

8 anos

Não

Sim

Não

10 anos

Não

16 anos

44 anos

$\mathrm{F}$

9.

Fonte: Dados da pesquisa, 2019.

Da análise dos dados, emergiram as seguintes categorias temáticas: orientações durante a consulta de pré-natal e percepção das enfermeiras sobre o cuidado ofertado durante o pré-natal. 


\section{Orientações durante a consulta de pré-natal}

A consulta de enfermagem é um importante instrumento, pois tem como finalidade garantir a extensão da cobertura e da melhoria do pré-natal por meio de ações promocionais e preventivas às gestantes e suas famílias. É essencial que o profissional, tenha competência técnica, sensibilidade e comunicação (RIOS CTF e VIEIRA NFC, 2007). Quando questionadas sobre os principais temas abordados durante as consultas de pré-natal, as enfermeiras demonstram a intenção de explanar que abordam de tudo, conforme a fala a seguir:

"Ah, é geral, eu abordo de tudo, desde vacinas, enxoval, a dúvida, oriento pra consulta odontológica, a vacina, já vou orientando mais próximo de nascer, 'ó tu vai fazer o teste do pezinho, teu bebê vai vir tal dia'..." (E1).

Em nenhuma das falas das entrevistadas o parto ou preparação para o parto foram referidos como os principais temas abordados durante a consulta de pré-natal, o que mostra que, para os profissionais, ainda é um tema que precisa ser mais abordado. O MS preconiza que, durante as consultas de pré-natal, a preparação para o parto, a orientação e o incentivo ao parto normal, o incentivo ao protagonismo da mulher, os sinais e os sintomas do parto e os benefícios legais que a mulher tem direito também devem ser abordados (BRASIL, 2012a).

Quando questionadas sobre os desafios da orientação em relação ao parto, as enfermeiras colocaram as seguintes questões:

"Aí, esse é complicado, porque elas têm... como que eu vou te dizer... elas ficam receosas. A gente orienta sempre, desde o início, todos os sintomas do trabalho de parto [...] a gente procura deixar elas bem orientadas pra que elas tenham consciência de se avaliar algum risco, né, e procurar o hospital qualquer coisa." (E1).

"Um dos desafios é o baixo grau de instrução, pouco estudo e as adolescentes, assim, não prestam atenção." (E1).

A enfermeira cita o baixo grau de instrução como uma questão de entendimento das orientações, juntamente com o fator idade (adolescência), referindo que as gestantes adolescentes não prestam atenção, pois ainda estão entendendo o processo. A maioria das gestações, nessa fase, embora possa ter sido desejada, não foi planejada. A adolescência está relacionada a maior ocorrência de parto prematuro (PP) e ao baixo peso ao nascer (BPN) e, consequentemente, a um pré-natal inadequado, o que implica em menor número de consultas e início tardio (MIRANDA FRD, 2013). As primigestas também foram citadas como desafios na abordagem do parto durante as consultas de pré-natal, conforme a fala a seguir:

"Tu sabe que assim, ó, as que são como a gente fala 'marinheira de primeira viagem' (risos), né, aí tem que conversar mais e mostrar a realidade... o parto, quando é essas que são primigesta, né, então tu tem que conversar um pouco mais, as que já são mãe de 2, 3, 4 filhos tem mais experiência, né, ai é mais fácil, sabe.." (E4).

A fala da enfermeira vai ao encontro do que preconiza o MS, o qual orienta a amparar a gestante na trajetória pré-natal, em especial na primeira gravidez, uma vez que a assistência, além de promover todos os cuidados e procedimentos que visam preservar a saúde da gestante e do feto, também deve incluir atividades de educação em saúde individual ou grupal que favoreçam hábitos saudáveis de vida, bem como o preparo para o parto e o puerpério (BRASIL, 2012a).

Uma das entrevistadas relatou não falar sobre o parto, ficando evidente que este, muitas vezes, é um assunto esquecido durante as consultas de pré-natal:

"Não falo quase, não falo, sei lá, acho que é uma deficiência minha, até porque, engraçado, parece que não faz parte do pré-natal, mas faz né (risos), e eu realmente não falo." (E2).

Da mesma forma, uma pesquisa realizada com gestantes sobre o parto, cujo tema é o pré-natal, apontou que a maior parte das gestantes relatou não ter tido conversas sobre parto na consulta médica até o momento 
da entrevista, sendo que as gestantes já estavam no terceiro trimestre de gestação (TOSTES NA e SEIDL EMF, 2016). Ressalta-se que as parturientes, quando informadas e esclarecidas sobre o momento do parto, tendem a ficar menos ansiosas e mais empoderadas e, geralmente, em razão disso, têm o processo de parto mais ameno e gratificante (RATTNER D, 2009; VIEIRA SM et al., 2011).

\section{Percepção das enfermeiras sobre o cuidado ofertado durante o pré-natal na ESF}

Percebe-se que, na maioria das falas, as enfermeiras refletiram sobre o seu saber/fazer no atendimento à gestante no pré-natal, sendo que três delas mencionaram o enfermeiro com muitas atribuições dentro da unidade, o que, por consequência, limita a qualidade do atendimento à gestante:

"Eu tenho muitas atribuições aqui dentro. Me sobrecarrego muito [...]." (E2).

"Tu tem que ter o apoio de todos, né, porque fica só pra enfermeira, cai só pra enfermeira. E ainda tem gente que acha que a gente não faz nada." (E4).

"Uma coisa assim não é só a enfermeira que existe, né, eu saí de licença, mas outra pessoa poderia ter assumido o grupo de gestantes" (E3).

O mesmo foi visto no estudo de Souza MG et al., (2013), realizado com 11 enfermeiros atuantes em ESF na cidade de Cuiabá, Mato Grosso do Sul. A responsabilização de forma desigual é vista como um problema, o que interfere no resultado do que entendem ser o trabalho específico do enfermeiro, provocando sobrecarga e limitando a qualidade do atendimento.

Uma das entrevistadas relatou que somente passou a prestar uma assistência adequada à puérpera quando teve seu filho, permitindo-nos verificar que ressignificou o seu saber/fazer, prestando atenção em um grupo antes deixado de lado durante as consultas:

" $E$ eu adorei agora, no momento que eu fiquei gestante e ganhei bebê, de dar mais atenção às puérperas, porque antes eu não dava atenção. Antes eu não perguntava muito, sabe, e agora não, eu vim com outro olhar." (E3).

Todas as enfermeiras relataram como ponto positivo a qualidade no processo de cuidado ofertado à gestante e às puérperas durante o pré-natal na ESF, como mostra o trecho a seguir:

"Eu acho que ponto positivo é a questão da orientação, tanto da enfermagem quanto da parte médica. As gurias técnicas de enfermagem também orientam bastante no momento da triagem, né." (E3).

Duas enfermeiras relataram fazer grupos de gestantes na unidade e uma disse estar organizando o primeiro grupo com a ajuda dos acadêmicos de Enfermagem, o que contribui para uma boa estratégia de educação em saúde:

"Grupo de gestantes a gente tá organizando o primeiro agora, com a ajuda dos acadêmicos." (E2).

"Grupo de gestante a gente faz." (E4).

"A gente faz grupo de gestante também, mas agora eu tava de licença, né, a gente ficou sem fazer os grupos." (E3).

O MS salienta a importância das atividades educativas realizadas junto às gestantes, seja em grupo ou individualmente, durante o pré-natal (BRASIL, 2012a). Dito isso, é possível afirmar que a utilização de novas estratégias educativas poderia possibilitar um maior esclarecimento sobre o próprio pré-natal, o trabalho de parto e o parto, haja vista que as mulheres podem desenvolver uma nova percepção de sua gestação, participando ativamente do processo de ensino-aprendizagem (ALVES ACP et al., 2013).

Sabe-se que a gestação traz consigo crenças e mitos disponibilizados por familiares, amigos e comunidade que cercam essa gestante. A educação em saúde durante o pré-natal, quando realizada por profissionais, tem o papel de desmistificar essas crendices. Para tanto, é importante que a família participe 
ativamente das consultas e dos grupos, juntamente com a gestante, e que recebam igualmente as informações repassadas. Quando questionadas sobre as fragilidades no cuidado a gestantes e puérperas no pré-natal na ESF, os relatos envolveram adesão ao pré-natal, rede de atenção à saúde, gerenciamento de equipe e abordagem no ciclo gravídico puerperal:

"Eu acho que a procura, adesão ao pré-natal, tu tem que tá muito em cima, tem que tá procurando, a grande maioria banaliza o atendimento. Eu acho que elas não dão tanta importância ao pré-natal." (E2).

Corroborando, em um estudo de coorte, no qual foram incluídas todas as usuárias do SUS que realizaram acompanhamento pré-natal em uma ESF na cidade de Porto Alegre (RS), foi identificada uma baixa adesão ao pré-natal, principalmente no primeiro trimestre, e frequência do número de consultas igual ou inferior a 6 durante o pré-natal. Isso colabora negativamente para um quadro de baixas orientações e precário preparo para o parto, a amamentação e o puerpério (HASS CN et al., 2013):

Uma das entrevistadas chamou atenção para seu relato sobre a abordagem ao ciclo gravídico puerperal e a dificuldade de lidar com as puérperas:

"Acho que as pessoas se mobilizam pra atender as gestantes, eu acho que é bem importante o pré-natal, é uma das coisas mais importantes da unidade, mas com a puérpera é bem complicado, é difícil elas marcarem o retorno em 15 dias e acaba passando, às vezes, no dia da consulta, eu pergunto como tá os lóquios, se a mama tá bem, essas coisas básicas, mas anotação e realmente parar para orientar a puérpera, olha, eu acho que fiz duas só." (E2).

Conforme o MS, o retorno da mulher e do recém-nascido à ESF deve ocorrer em torno de cinco a dez dias após o parto. Esse retorno deve ser estimulado durante as consultas de pré-natal, na maternidade e também pelos ACSs durante a visita domiciliar. O objetivo do retorno da mulher é avaliar o seu estado de saúde e do recém-nascido, orientar quanto à amamentação e aos cuidados básicos com o bebê e avaliar o convívio entre o binômio, a fim de prevenir situações de morbidade e mortalidade materna e neonatal, uma vez que boa parte dessas situações ocorre na primeira semana após o nascimento do bebê (BRASIL, 2012a).

\section{CONSIDERAÇÕES FINAIS}

A orientação sobre o momento do parto não apareceu entre os temas abordados durante a consulta de pré-natal, e algumas reconheceram que não lembram do parto como um tema a ser utilizado nas consultas de pré-natal. Torna-se evidente a necessidade de educação permanente desses profissionais, bem como documentos e cartilhas que auxiliem nas orientações em relação ao parto durante as consultas de pré-natal e em grupos de gestantes para que as gestantes não cheguem no momento do parto sem informação. Reconhece-se que novos estudos precisam ser realizados com o intuito de ampliar e fortalecer o trabalho do enfermeiro frente ao pré-natal na ESF, principalmente relacionados às orientações para o momento do parto.

\section{REFERÊNCIAS}

1. ALVES ACP, et al. Aplicação de tecnologia leve no pré-natal: um enfoque na percepção das gestantes. Rev. enferm. UERJ, Rio de Janeiro, 2013; $21(5)$ : 648-53.

2. ARAUJO SM, et al. A Importância do pré-natal e a assistência de enfermagem. Revista Eletrônica de Ciências, 2010; 3(2): 61-68.

3. BARDIN L. Análise de conteúdo. Lisboa: Edições 70, 2011.

4. BRASIL. Ministério da saúde. Conselho Nacional de Saúde. Resolução no 466, de 12 de dezembro de 2012. Aprova diretrizes e normas regulamentadoras de pesquisa envolvendo seres humanos. Brasília: Ministério da Saúde, $2012 b$.

5. BRASIL. Ministério da Saúde. Portaria oㅜ 2.436, de 21 de setembro de 2017. Aprova a Política Nacional de Atenção Básica, estabelecendo a revisão de diretrizes para a organização da Atenção Básica, no âmbito do Sistema Único de Saúde (SUS). Brasília: Diário Oficial da União, 2017. 
6. BRASIL. Ministério da Saúde. Secretaria de Atenção à Saúde. Departamento de Atenção Básica. Atenção ao prénatal de baixo risco/Ministério da Saúde. Secretaria de Atenção à Saúde. Departamento de Atenção Básica. 1. ed. Brasília: Ministério da Saúde, 2012a.

7. BRASIL. Ministério da Saúde. Secretaria de Atenção à Saúde. Departamento de Ações Programáticas Estratégicas. Relatório de gestão 2003 a 2006: Política Nacional de Atenção Integral à Saúde da Mulher/Ministério da Saúde. Brasília: Ministério da Saúde, 2007.

8. HASS CN, TEIXEIRA LB, BEGHETTO MG. Adequabilidade da assistência pré-natal em uma Estratégia de Saúde da Família de Porto Alegre, RS. Rev. Gaúcha Enfermagem, 2013; 34(3): 22-30.

9. MIRANDA FRD, et al. Pré-natal na adolescência: uma revisão crítica. Adolescência \& Saúde, Rio de Janeiro, 2013; 10(1): 43-50.

10. RAMOS ASMB, et al. A assistência pré-natal prestada pelo enfermeiro sob a ótica das gestantes. Revista Interdisciplinar, 2018; 11(2): 87-96.

11. RAGAGNIN MV, et al. Abordagem da equipe de enfermagem acerca do parto humanizado no pré-natal: uma visão narrativa. Rev. Fund Care Online, 2017; 9(4): 1177-1182.

12. RATTNER D. Humanização na atenção a nascimentos e partos: Breve referencial teórico. Interface: Comunicação, Saúde, Educação, 2009; 13(1), 595-602.

13. RIOS CTF, VIEIRA NFC. Ações educativas no pré-natal: reflexão sobre a consulta de enfermagem como um espaço para educação em saúde. Ciência \& Saúde Coletiva, 2007; 12(2): 477-486.

14. SILVA MB, MONTEIRO PS. Adequação do pré-natal em gestantes atendidas na Estratégia de Saúde da Família em Palmas-TO, 2009. Com. Ciências Saúde, 2010; 21(1): 21-30.

15. SOUZA MG, MANDU ENT, ELIAS AN. Percepções de enfermeiros sobre seu trabalho na estratégia saúde da família. Texto Contexto Enfermagem, Florianópolis, 2013; 22(3):772-9.

16. TOSTES NA, SEIDL EMF. Expectativas de Gestantes sobre o Parto e suas Percepções acerca da Preparação para o Parto. Temas em Psicologia, Ribeirão Preto, 2016; 24(2): 681-693.

17. VIEIRA SM, et al. Percepção das puérperas sobre a assistência prestada pela equipe de saúde no pré-natal. Texto e Contexto Enfermagem, Florianópolis, 2011; 20(esp): 255-262. 